\title{
Investigating for the aetiology of Cushing's syndrome: where we can go wrong
}

\author{
M I Weerakkody ${ }^{1}$, P Katulanda ${ }^{2}$, S Perera ${ }^{3}$ \\ Sri Lanka Journal of Diabetes, Endocrinology and Metabolism 2012; 2: 114-117
}

\begin{abstract}
The diagnosis of Cushing's syndrome as well as delineating its aetiology can be often challenging, especially in the mild cases with atypical features. Although the combined clinical features such as the age, gender and the severity of the hypercortisolism can usually help to differentiate between pituitary ACTH hyper secretion from an ectopic ACTH source, this may sometimes be inaccurate. The presence of caveats in most biochemical testing and imaging modalities as well as the relative unavailability of certain dynamic tests and catheter based studies can contribute to the dilemma.
\end{abstract}

\section{Introduction}

Differentiation between the two main aetiologies of ACTH dependent Cushing's syndrome, an ACTHproducing pituitary adenoma and an ectopic ACTHproducing tumor, can be challenging when an obvious source of ACTH hypersecretion is absent. Cushing's disease accounts for about $90-95 \%$ of the cases of ACTH dependent Cushing's syndrome. Although many patients have clinical and/or biochemical features suggestive of one of the two diagnoses, no one biochemical test or imaging procedure correctly identifies the source of ACTH hypersecretion in all cases. The evaluation of ACTHdependent Cushing's syndrome has been greatly improved by the availability of bilateral inferior petrosal sinus sampling (BIPSS). Here we report a case of a patient with Cushing's syndrome, whose pituitary source of ACTH secretion was ultimately diagnosed with BIPSS after extensive investigation with other methods for more than two years.

\section{Case report}

Mr C, a previously healthy businessman was detected to be hypertensive at the age of 35 years and was started on Losartan K 50 mg twice daily. However, his blood pressure remained uncontrolled despite good compliance for treatment, generally above 160/110. About one year later $\mathrm{Mr} \mathrm{C}$ began to notice an increase in bodyweight and change in facial appearance. He also noticed skin petechiae, mainly in the upper and lower limbs. He had not noticed a recent increase in skin pigmentation. On examination, he had truncal obesity with a body mass index of $28.1 \mathrm{~kg} / \mathrm{m}^{2}$. He also had dorso-scapular fat deposition, facial fullness, acne and facial plethora. His blood pressure was 160/100 while on medication and the fundoscopy revealed grade 2 hypertensive retinopathy. There was clinical evidence of a grade 4 proximal myopathy of all limbs. He also had enlargement of the thyroid gland with 2 firm nodules over the right lobe.

His basic investigations revealed a persistently low serum potassium, usually in the range of 3-3.5 mmol/l. The rest of the haematological and biochemical investigations were normal. Since the clinical features were suggestive of Cushing's syndrome, a low dose dexamethasone suppression test was performed which showed an unsuppressed cortisol value of $327 \mathrm{nmol} / \mathrm{l}$ (normal $<50$ ) at 48 hours. His 24 hour urinary free cortisol was 683 mmol (0-283). Therefore Cushing's syndrome was biochemically confirmed. His serum ACTH value was unsuppressed at $49.7 \mathrm{pg} / \mathrm{ml}(0-46)$ suggesting that this was ACTH dependent disease. A pituitary MRI was performed as pituitary ACTH secretion was responsible for $90-95 \%$ of ACTH dependent Cushing's syndrome, which failed to reveal a lesion. A gadolinium enhanced dynamic pituitary imaging, which has a higher sensitivity for pituitary lesions was also negative. We did not have facilities in Sri Lanka to perform bilateral inferior petrosal venous sinus (BIPSS) sampling at that time. A high dose Dexamethasone suppression test failed to suppress cortisol to more than $50 \%$ of the basal value suggesting that this was more likely to be ectopic ACTH secretion. The fact that this was a male patient and the presence of persistent hypokalemia were also supportive of this. We performed a CT scan chest and abdomen to identify a source of ACTH secretion which showed multiple nodules in the right lobe of the thyroid gland with mixed echo

${ }^{1}$ Senior Registrar, Endocrine and Diabetes Unit, National Hospital of Sri Lanka, Colombo, ${ }^{2}$ Senior Lecturer and Consultant Endocrinologist, Department of Clinical Medicine, Faculty of Medicine, Colombo, ${ }^{3}$ Consultant Neurosurgeon, The Central Hospital, Colombo. 
pattern. Thymic area showed a few foci of soft tissue density material with no definitive mass. The possibility of a thyroid or thymic carcinoid secreting ACTH was considered but needed to be confirmed further with investigations. We planned to carry out further investigations to identify an occult ACTH secreting tumour while managing the patient medically to minimize the adverse effects of persistent hypercortisolism. Mr C was started on oral metyrapone $250 \mathrm{mg}$ bid and oral ketoconazole $100 \mathrm{mg}$ bid which brought his cortisol levels to within normal limits after a few weeks.

A fine needle aspiration biopsy of the thyroid nodules revealed a colloid storing goiter with degenerative changes only. Colonoscopy and bronchoscopy failed to reveal any lesions suggestive of carcinoids. As the possibility of a carcinoid, which is known to cause low grade Cushing's syndrome due to ectopic ACTH secretion, was high, we performed several biochemical tests which are tumour markers of carcinods. The 24 hour urinary 5 hydroxy indolacetic acid (5HIAA) and serum Chromogranin A levels were normal. A 99 m In-Octreotide scan was performed to detect any sites of occult neuro-endocrine activity with ACTH secretion; this was normal.

As the patient's problem was still unresolved and as the patient was on longterm treatment with drugs with a number of possible adverse effects, we contemplated a combined thyroidectomy and a thymectomy for the only possible sites of ectopic ACTH secretion that we have identified. However, as bilateral inferior petrosal sinus sampling (BIPSS) became available at the National Hospital of Sri Lanka we planned for that procedure considering the fact that $90-95 \%$ of ACTH dependent Cushing's syndrome was due to pituitary disease. We also included mediastinal vein sampling as we were also considering thyroid or the thymus as the possible sites of ACTH secretion.

\title{
Results of BIPSS
}

\author{
Inferior petrosal sinus $\mathbf{A C T H}$ Right: $\mathbf{6 1 0} \mathrm{pg} / \mathrm{dl}$ \\ Left : $191 \mathrm{pg} / \mathrm{dl}$
}

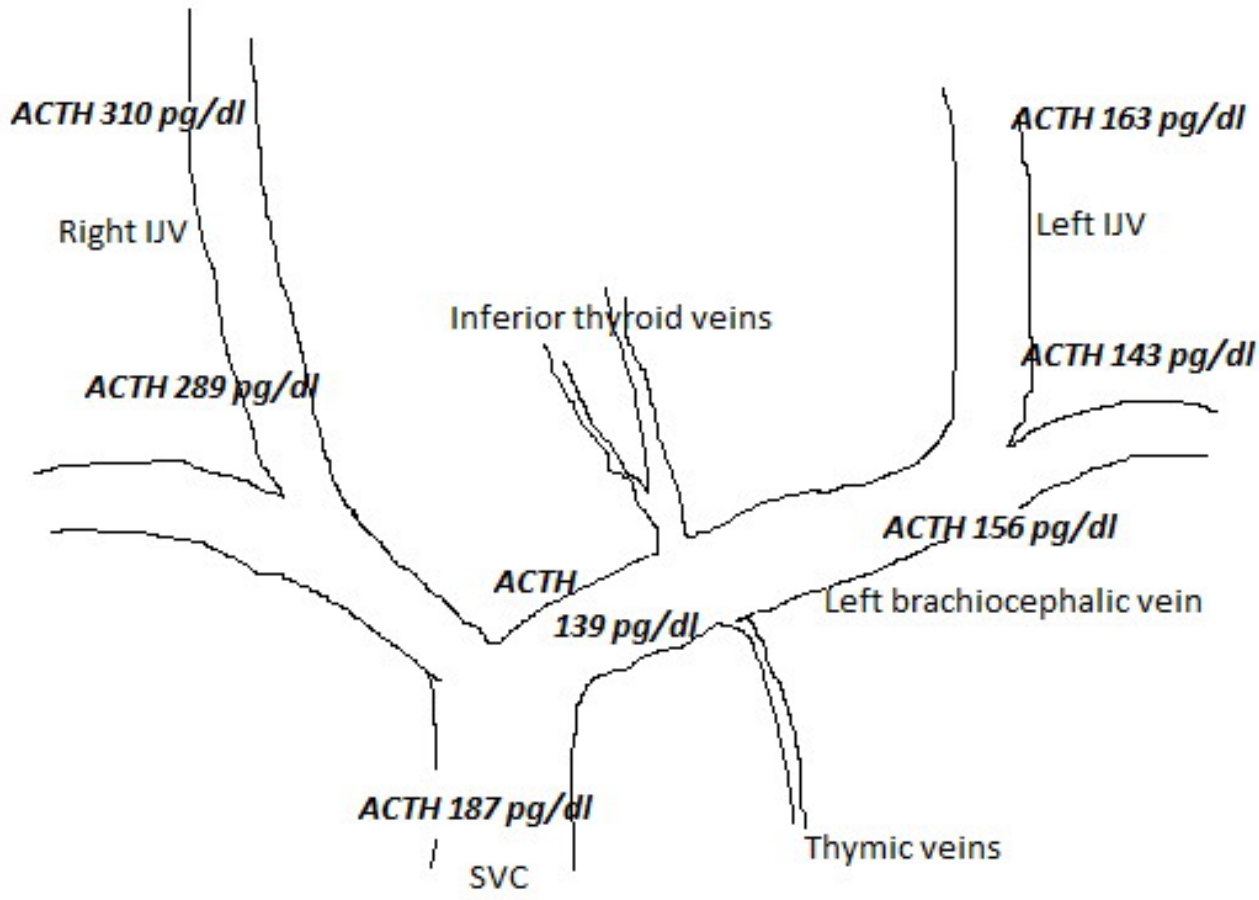

Femoral ACTH $126 \mathrm{pg} / \mathrm{dl}$ 
The ACTH values of the right inferior petrosal sinus was about six times higher than the other values of the periphery (femoral vein); a central to peripheral gradient of more than 2 in an unstimulated test is considered significant with a diagnostic sensitivity of nearly $100 \%$ (1). Therefore venous sampling clearly identified the site of ACTH secretion as the pituitary as opposed to an ectopic site, most likely involving the the right side of the pituitary gland.

The immediately pre operative MRI scan of the pituitary identified an ill defined $4 \mathrm{~mm} \times 3 \mathrm{~mm}$ non enhancing mass in the right lobe of the pituitary suggestive of an adenoma. The patient underwent transphenoidal excision of the pituitary with an uneventful post operative period. The pathological specimen consisted of a $4 \times 4 \mathrm{~mm}$ well defined mass lesion with cells containing round nuclei, dispersed chromatin and eosinophilic granular cytoplasm on histology. The serum cortisol levels were $<50 \mathrm{nmol} / 1$ post operatively, indicating a cure from the disease. Most of his symptoms improved following surgery, and the blood pressure normalized.

\section{Discussion}

This case illustrates the importance of bilateral inferior petrosal sinus sampling in the diagnosis of the aetiology of ACTH dependent Cushing's syndrome.

The vast majority (90-95\%) of patients with ACTH dependent Cushing's syndrome have a pituitary corticotroph microadenoma (1). Because the pretest probability of Cushing's disease is so high, any differential diagnostic test must be very accurate. Simple clinical measures have been shown to have good predictive value in establishing the presence of Cushing's disease. A woman with mild to moderate hypercortisolism, a normal or slightly elevated plasma ACTH, and normokalemia has at least a 95\% likelihood of having Cushing's disease. In contrast, a patient with overt hypercortisolism, hypokalemia, and marked elevations of plasma ACTH may be more likely to have an occult ectopic ACTH-secreting tumor (2). Our patient was a young man with persistent hypokalemia, unusual features for pituitary ACTH production, which made us consider ectopic ACTH production as a possible aetiology. Although ectopic ACTH production would be expected to have higher values of ACTH production and overt hypercortisolism than in our patient, low grade Cushing's is still possible with certain ectopic ACTH sources like carcinoids. Studies have shown that certain neuroendocrine tumours such as bronchial and thymic carcinoids can present with covert ectopic ACTH secreting syndrome and may actually have marginally high ACTH levels resembling pituitary disease (3).

Pituitary imaging with MRI is the initial study required when investigating for a cause for ACTH dependent Cushing's syndrome. Unfortunately, as in our patient,
$40-50 \%$ of corticotroph microadenomas causing Cushing's syndrome in adults are not visible with this technique (4). The use of dynamic MRI (with iv gadolinium) with spoiled gradient sequences may increase the sensitivity (5). It is also important repeat the MRI after a reasonable time interval if the initial scans were negative but the patient remains symptomatic. Because as hypercortisolaemia evolves, it is possible for the repeat scans to demonstrate microadenomas which were undetected previously due to the minute size, just as it occurred in our patient. Although there is a consensus that the presence of a pituitary lesion greater than $6 \mathrm{~mm}$ in a patient with a classical presentation and concordant dynamic results provides a definite diagnosis of Cushing's disease (6), further evaluation in the absence of a pituitary lesion is not so clearly defined.

There are a number of non invasive tests that can be used concurrently when the pituitary imaging is negative, but none of them have the diagnostic accuracy alone to differentiate the source of ACTH production. The high dose dexamethasone suppression test, using the criterion of a cortisol suppression of $>50 \%$ of the baseline level after high-dose dexamethasone has a sensitivity and specificity of 88 and 57\% (7). Low grade Cushing's, as in a carcinoid syndrome can give a false negative result and overt hypercortisolism in Cushing's disease can sometimes give a false positive value.

Although the CRH stimulation test is considered the most reliable dynamic noninvasive test for the differential diagnosis of ACTH-dependent Cushing's syndrome, this test cannot make a $100 \%$ differentiation between pituitary and ectopic causes. From literature data, the sensitivity and specificity using ACTH criteria is approximately $90 \%$. However, there is yet no consensus on the criteria for interpreting the test as positive. Variability in interpretation depends on the type of CRH used, the weight based or fixed dose of $\mathrm{CRH}$, and the wide range of response parameters (8). CRH is not currently available in Sri Lanka.

On the contrary, BIPSS is considered the gold standard for establishing the origin of ACTH secretion. Given the fact that $95 \%$ of the patients with ACTH dependent Cushing's syndrome would harbour a pituitary lesion, BIPSS is recommended to be done in patients like ours who either have a negative MRI or have discordant imaging and biochemical results (5). Furthermore, ectopic ACTH secreting tumours are only responsible for about $5-10 \%$ of the ACTH dependent Cushing's syndrome. Localization of these tumours is also often challenging and extremely difficult, imaging correctly identifies the tumour at first investigation in only $65 \%$ of cases. In up to $30 \%$ of cases, the tumour remains occult for a long time after diagnosis of Cushing's syndrome (9). Although tumour markers such as plasma calcitonin, gastrin, glucagon, or somatostatin may often be elevated in patients with ectopic ACTH syndrome, they are rarely helpful in identifying the source of the neoplasm (10). Somatostatin receptor scintigraphy, such as the Octreotide 
scan which was used in our patient, may not sometimes detect the ACTH secreting neuro-endocrine tumours, due to inadequate resolution of the scans to detect these small lesions (1). Most of these tests are very expensive and not freely available in Sri Lanka. Therefore although BIPSS is an invasive test that is only performed in certain tertiary centres currently, it should be performed in all patients prior to embarking on a tedious search for a source of ectopic ACTH secretion. Extensive imaging without performing BIPSS may result in false-positive studies and lead to surgical misadventures.

This case illustrates the difficulties that are sometimes encountered when investigating for the aetiology of ACTH dependent Cushing's syndrome and the important role of BIPSS in the investigation pathway. It also highlights the need for making BIPSS a more widely available procedure in a resource poor setting like ours, where the performance of a multitude of other tests on each patient would not be feasible.

\section{Abbreviations}

ACTH - adrenocorticotropic hormone, CRH corticotrophin releasing hormone, IJV - internal jugular vein, BIPSS - bilateral inferior petrosal sinus sampling.

\section{References}

1. Findling JW, Raff H. Clinical review: Cushing's syndrome: important issues in diagnosis and management $J$ of Clin Endocrinol Metab 2006; 91(150): 3746-53.

2. Aron DC, Raff H, Findling JW. Effectiveness versus efficacy: the limited value in clinical practice of high dose dexamethasone suppression testing in the differential diagnosis of adrenocorticotropin-dependent Cushing's syndrome. J Clin Endocrinol Metab 1997; 82: 1780-5.

3. Isidori AM, Kaltsas GA, Pozza C. Ectopic adreno- corticotropin syndrome: clinical features, diagnosis, management, and long-term follow-up. J of Clin Endocrinol Metab 2006; 91(2): 371-377.

4. Tabarin A, Laurent F, Catargi B, Olivier-Puel F, et al 1998. Comparative evaluation of conventional and dynamic magnetic resonance imaging of the pituitary gland for the diagnosis of Cushing's disease. Clin Endocrinol (Oxf) 49: 293-300.

5. Patonas N, Bulakbasi N, Stratakis CA, Lafferty A, et al. Spoiled gradient recalled acquisition in the steady state technique is superior to conventional postcontrast spin echo technique for magnetic resonance imaging detection of adrenocorticotrophin-secreting pituitary tumors. $J$ Clin Endocrinol Metab 2003; 88: 1565-9.

6. Testa, N Albiger G, Occhi F Sanguin. The usefulness of combined biochemical tests in the diagnosis of Cushing's disease with negative pituitary magnetic resonance imaging. European Journal of Endocrinology 2007; 156: 241-248

7. Dichek HL, Nieman LK, Oldfield EH, Pass HI, et al. A comparison of the standard high dose dexamethasone suppression test and the overnight $8 \mathrm{mg}$ dexamethasone suppression test for the differential diagnosis of adrenocorticotropin-dependent Cushing's syndrome. $J$ of Clin Endocrinology Metab 1993; 78: 418-22.

8. Boscaro M, Arnaldi G. Approach to the patient with possible Cushing's syndrome. J of Clinl Endocrin Metab 2009; 94(9): 3121-31.

9. Ilias I, Torpy DJ, Pacak K, Mullen N, et al. Cushing's syndrome due to ectopic corticotropin secretion: twenty years' experience at the National Institute of Health. J Clin Endocrinol Metab 2005; 90: 4955-62.

10. Isidori AM, Kaltsas GA, Pozza C, Frajese V, et al. The ectopic adrenocorticotropin syndrome: clinical features, diagnosis, management, and long term follow-up. J Clin Endocrinol Metab 2005; 91: 371-7. 